\title{
A conserved role for sleep in supporting Spatial Learning in Drosophila
}

\author{
Krishna Melnattur ${ }^{1, \bullet}$, Leonie Kirszenblat ${ }^{2,3}$, Ellen Morgan ${ }^{1, \bullet}$, \\ Valentin Militchin ${ }^{4}$, Blake Sakran ${ }^{1}$, Denis English ${ }^{1}$, Rushi Patel ${ }^{1}$, \\ Dorothy Chan ${ }^{1}$, Bruno van Swinderen ${ }^{2}$ and Paul J. Shaw ${ }^{1, *, \bullet}$
}

${ }^{1}$ Department of Neuroscience, Washington University School of Medicine, St Louis, MO, ${ }^{2}$ Queensland Brain Institute, The University of Queensland, St Lucia, QLD, Australia, ${ }^{3}$ RIKEN Center for Brain Science, Wako, Saitama, Japan, ${ }^{4}$ Department of Otolaryngology, Washington University School of Medicine, St Louis, MO

*Corresponding author. Paul J Shaw, Department of Neuroscience, Washington University School of Medicine, Campus Box 8108, 660 South Euclid Avenue, St Louis, MO. Email: shawp@wustl.edu.

\begin{abstract}
Sleep loss and aging impair hippocampus-dependent Spatial Learning in mammalian systems. Here we use the fly Drosophila melanogaster to investigate the relationship between sleep and Spatial Learning in healthy and impaired flies. The Spatial Learning assay is modeled after the Morris Water Maze. The assay uses a "thermal maze" consisting of a $5 \times 5$ grid of Peltier plates maintained at $36-37^{\circ} \mathrm{C}$ and a visual panorama. The first trial begins when a single tile that is associated with a specific visual cue is cooled to $25^{\circ} \mathrm{C}$. For subsequent trials, the cold tile is heated, the visual panorama is rotated and the flies must find the new cold tile by remembering its association with the visual cue. Significant learning was observed with two different wild-type strains-Cs and $2 \mathrm{U}$, validating our design. Sleep deprivation prior to training impaired Spatial Learning. Learning was also impaired in the classic learning mutant rutabaga (rut); enhancing sleep restored learning to rut mutants. Further, we found that flies exhibited a dramatic age-dependent cognitive decline in Spatial Learning starting at 20-24 days of age. These impairments could be reversed by enhancing sleep. Finally, we find that Spatial Learning requires dopaminergic signaling and that enhancing dopaminergic signaling in aged flies restored learning. Our results are consistent with the impairments seen in rodents and humans. These results thus demonstrate a critical conserved role for sleep in supporting Spatial Learning, and suggest potential avenues for therapeutic intervention during aging.
\end{abstract}

\section{Statement of Significance}

We have studied the relationship between sleep and plasticity using a Drosophila learning assay modified after the Morris Water Maze. Using this assay, we find that sleep loss impairs Spatial Learning. As in mammals, flies exhibited age-dependent Spatial Learning impairments. Importantly, the age-dependent impairments were reversed by enhancing sleep. Interestingly, our results mirror studies on hippocampus-dependent memories in rodents and humans. Thus, our data describe an evolutionarily conserved role for sleep in regulating Spatial Learning. They also support augmenting sleep as a therapeutic strategy to ameliorate learning impairments.

Key words: sleep; Drosophila; Spatial Learning; aging; plasticity 


\section{Introduction}

While a precise function of sleep remains unclear [1], many lines of evidence point to a pivotal role for sleep in supporting learning and memory [2-4]. Further, cognitive impairments associated with aging and neurodegenerative disorders are associated with defects in sleep [5, 6]. Understanding how sleep benefits neural function thus has the potential to not only reveal novel insights into brain function, but also to suggest avenues for therapeutic intervention in animals whose nervous systems are challenged by aging or neurodegenerative diseases. In humans, sleep supports many kinds of memories [7-10]. However, declarative memories-memories of experiences (episodic memory) and memories of facts (semantic memory), appear to particularly benefit from sleep [11]. Importantly, sleep supports both the encoding of new information $[12,13]$ and the consolidation of learned information into a memory [11]. Further, defects in encoding new declarative memories such as new facts or names are a common feature of cognitive decline in aging and degenerative disease $[5,14]$. Studying declarative memories in animal models remains challenging. However, rodent Spatial Learning and human declarative memories share common cellular substrates and computations leading to the proposal that rodent Spatial Learning is an evolutionary precursor of human episodic memory $[15,16]$. A Spatial Learning assay has been described in flies $[17,18]$. Here we have adapted this Spatial Learning assay for sleep-plasticity studies, and use it to investigate the effects of enhancing sleep on learning impairments resulting from aging and the classic memory mutant rutabaga (rut).

\section{Methods}

Flies

Flies were cultured at $25^{\circ} \mathrm{C}$ at $\sim 50 \%$ relative humidity, and reared on a standard yeast, corn syrup, molasses, and agar diet while being maintained on a $12 \mathrm{~h}$ light:12 h dark cycle. Female flies were used as subjects in most experiments except for the experiment with rut $^{2080}$ flies in Figure 3, where male flies were used, as female rut ${ }^{2080}$ flies do not exhibit learning deficits in the Aversive Phototaxis Suppression Assay. For aging experiments, flies were maintained in vials in groups of 10 . We evaluated potential time of day effects on Spatial Learning, but did not observe any changes in performance over the course of the day. Accordingly, most learning experiments were performed in the afternoon between 12:00 pm and 05:00 pm, except for the sleep deprivation experiments which were performed in the morning following overnight sleep deprivation.

\section{Fly strains}

Cs flies were obtained from T. Zars (Univ. of Missouri). 2U and rut $^{2080} / F M 7 c$ flies were gifts of J. Dubnau (Stonybrook University, NY). UAS Kir2.1EGFP (homozygous viable 3rd chromosome insert) was a gift of R. Baines (Manchester). THGAL4 (RRID: BDSC_8848, 3rd chromosome insert), R15B07GAL4 (RRID:BDSC_48676), R23E10-GAL4 (RRID:BDSC_49032), tubPGAL80 ${ }^{\text {ts }}$ (RRID:BDSC_7017, 3rd chromosome insert), and UAS NaChBacEGFP 4 (RRID:BDSC_9466, homozygous viable 2nd chromosome insert) flies were obtained from the Bloomington
Drosophila Stock Center. dumb ${ }^{2}$ (Dop1R1 $1^{\text {fo2676 }}$ ) flies were obtained the Exelexis collection. The UAS NaChBacEGFP, tubPGAL80 ${ }^{\text {ts }}$, and UAS Kir2.1EGFP stocks were backcrossed to a reference yw strain for five generations. We evaluated several strains to determine if they were wild-type for a number of parameters including sleep, sleep homeostasis, social enrichment, and learning in the Aversive Phototaxis Suppression and courtship conditioning assays. The yw strain is wild-type for these and other phenotypes and we used it as necessary.

\section{Drug feeding}

Gaboxadol (Sigma-Aldrich, St Louis, MO) was fed to flies at a concentration of $0.1 \mathrm{mg} / \mathrm{mL}$ dissolved in standard fly food as previously described [19]. 3IY (Sigma-Aldrich) was administered in the food at $10 \mathrm{mg} / \mathrm{mL}$, and L-Dopa (Sigma-Aldrich) was dissolved in the food at $5 \mathrm{mg} / \mathrm{mL}$ as per established protocols [20]. Flies were maintained on food with the appropriate drugs for 2 days prior to testing.

\section{Sleep}

Sleep was measured using protocols previously described [21]. Briefly, individual flies were aspirated into $65 \mathrm{~mm}$ glass tubes with standard fly food at one end, and their locomotor activity was continuously monitored using the Drosophila Activity Monitoring (DAM) System (Trikinetics, Waltham, MA). Locomotor activity was binned in 1 min intervals; sleep, defined as 5 min of inactivity, was computed using custom Excel scripts. In sleep plots, sleep in $\mathrm{min} / \mathrm{h}$ is plotted as a function of Zeitgeber Time (ZT). ZTO represents the beginning of the fly's subjective day (lights on) and ZT12 represents the transition from lights on to lights off.

\section{Sleep homeostasis}

4-7 days old female flies were placed in DAM tubes and their sleep was recorded for 2 days to establish a baseline. Flies were then sleep deprived for $12 \mathrm{~h}$ during the dark phase (ZT12-ZT0) by placing DAM monitors in the Sleep Nullifying APparatus using procedures previously described [20]. For each individual fly, the difference in sleep time in the two recovery days and the baseline day was calculated as the sleep gained/lost. Sleep rebound was only evaluated to ensure that the sleep deprivation experiment was effective and that flies had been successfully kept awake. Learning was evaluated in an independent cohort of flies immediately following overnight sleep deprivation.

\section{Visual learning protocol}

We constructed a visual place learning assay modeled on the classic Morris Water Maze (Figure 1) [17, 18]. Our assay uses a "thermal maze" consisting of a grid of Peltier plates maintained at $36-37^{\circ} \mathrm{C}$ (which is aversive to flies), and a distal visual panorama. One of four tiles can be cooled to $\sim 25^{\circ} \mathrm{C}$. The visual panorama was arranged such that the edge between the horizontal bar panel and the vertical bar panel marked the cool spot. The experimental protocol consisted of ten 3-min training trials with a 1 min break between trials. Software written in processing 


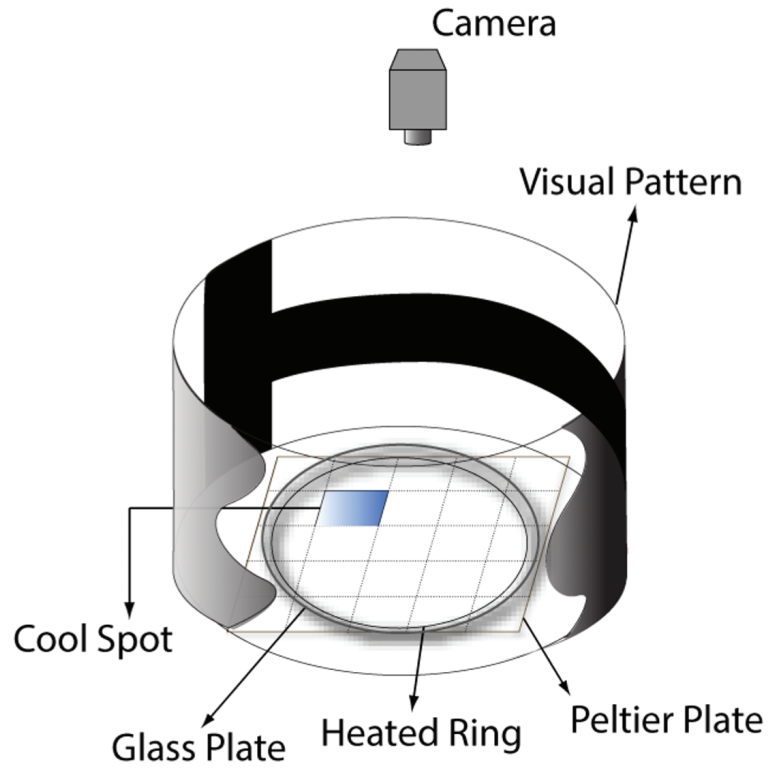

Figure 1. Spatial learning apparatus. The floor of the apparatus is made up of a $5 \times 5$ grid of Peltier plates, which are maintained at a temperature of $36-37^{\circ} \mathrm{C}$ (which is aversive to flies). The first trial begins when a single fly in placed into the apparatus and one of the tiles is cooled to $25^{\circ} \mathrm{C}$ via an Arduino Uno controller (not shown). Distal visual cues mark the cool spot. In subsequent trials, the location of the cool spot and the distal visual cues move in tandem such that the fly learns to associate the visual cue with the location of the cool spot. The location of the flies is monitored using a camera (see methods for details).

(Processing Foundation) generated a random list of $10 \mathrm{cool}$ spot locations. The first trial begins when a single tile that is associated with the visual cue is cooled to $25^{\circ} \mathrm{C}$. For subsequent trials, in the coupled condition, the previously cold tile is heated, and the visual panorama is rotated such that the flies must find the new cold tile by remembering its association with the visual cue. In the uncoupled condition, cool spot locations were changed between trials but the distal visual cues remained fixed to the location in the first trial.

To evaluate learning, an individual fly is placed into the apparatus and the time to find the cool spot is calculated. Individual flies remained in the arena for the duration of the experiment (10 trials). Experimenters were blinded to condition/genotype. Learning during subsequent trials, expressed as the time to target, was normalized to the time to find the cold spot in the first trial. Further, a learning index was computed as the percentage change in relative time to target as: Learning Index $=(1-$ [average relative time to target in trials $9 \& 10])^{*} 100$.

\section{Construction of arena}

The design of our visual place learning apparatus and visual panorama was adapted from previously described designs [17, 18]. The floor of the apparatus constituted a thermal maze and was composed of twenty-five $40 \mathrm{~mm} \times 40 \mathrm{~mm}$ Peltier devices (Custom Thermoelectric \#12711-5L31-06CQ Bishopville, MD) arranged in a $5 \times 5$ grid. This Peltier grid was covered by white masking tape to create a uniform surface. The grid was connected in five groups which were soldered in series in each group to reduce the difference in temperature between the first and the last components. Four Peltier tiles of the central 9 tiles can change their state independently from cooling to heating by relays. The temperature is measured by a thermocouple and sampled with an Arduino Uno. The micro-controller controls the temperature with a power supply by changing the constant current going through the Peltier elements. The Arduino Uno also controls which Peltier device to change from a heating to cooling state. The Peltier array was maintained at $36-37^{\circ} \mathrm{C}$ except for the four tiles which could be selectively cooled to $\sim 25^{\circ} \mathrm{C}$.

Flies were confined to this arena by means of a heated $3 \mathrm{~mm}$ high, $200 \mathrm{~mm}$ diameter aluminum ring that circumscribed the arena. The ring was connected by means of insulated wire to a power supply (BK Precision 1685B), which ensured that the ring was heated to $50^{\circ} \mathrm{C}$, thus keeping the flies away from the walls. A glass dish coated with the siliconizing reagent Sigmacote (Sigma-Aldrich) was placed on top of the ring. The distal visual cues used for place learning consisted of one panel of alternating black \& white vertical bars, one panel of alternating black \& white horizontal bars, and one panel of alternating black \& white angled bars printed on white paper and held together with clips. When viewed from the arena's center the width of each bar spanned $15^{\circ}$. The arena was illuminated with white light, and the fly's position was recorded with a webcam (Logitech 270).

\section{Heat avoidance}

Flies were confined to a chamber spanning the dimensions of two Peltier tiles using Lego bricks (Billund, Denmark). One of the tiles was maintained at $36-37^{\circ} \mathrm{C}$ and the other at $25^{\circ} \mathrm{C}$. The walls of the chamber were coated with Sigmacote (Sigma-Aldrich) to prevent flies from climbing on the sides. Flies thus had to choose between the hot side and the cool side. The heat avoidance index was calculated as the fraction of time flies spent on the cool side in a 3-min trial. Wild-type flies typically spent $\sim 80 \%-$ $90 \%$ of the time on the cool side. As a control, we also tested flies when both Peltier tiles were at the same (hot) temperature. In this condition, flies did not display a preference for either side (data not shown).

\section{Optomotor}

Flies had their wings clipped on $\mathrm{CO}_{2}$, at least 2 days prior to the experiment. During the experiment, flies walked freely on a round platform, $86 \mathrm{~mm}$ in diameter, surrounded by a waterfilled moat to prevent escape. Experiments using moving gratings were conducted with clockwise and anticlockwise gratings for $1.5 \mathrm{~min}$ each. Independent flies were used for each 3-min experiment. The temperature of the arena was $24-26^{\circ} \mathrm{C}$ during experiments. The walls of the arena consisted of 6 LED panels that formed a hexagon surrounding the moat $(29 \mathrm{~cm}$ diameter, $16 \mathrm{~cm}$ height), and onto which the visual stimuli were presented. Each LED panel comprised 1,024 individual LED units (32 rows by 32 columns) and was computer controlled with LED Studio software (Shenzen Sinorad, Medical Electronics, Shenzen, China). A camera (SONY Hi Resolution Colour Video Camera CCD-IRIS SSC-374) placed above the arena was used to detect the fly's movement on the platform at 30 frames/ s, and open-source tracking software was used to record the position of the fly [22]. All visual stimuli were created in VisionEgg software [23] written in Python programming language. The refresh rate was $200 \mathrm{~Hz}$. The luminance of the LED panels was approximately 770 Lux, 
reaching 550 Lux at the center of the arena. A grating of alternating cyan and black stripes was rotated in either direction (1.5 min each), with a temporal frequency of $3 \mathrm{~Hz}$ and spatial frequency 0.083 cycles/degree. Analyses were performed using CeTran (3.4) software [22], as well as custom made scripts in $R$ programming language. For optomotor responses, the angular velocity (turning angle/s) in the direction of the moving grating was calculated.

\section{Statistical analysis}

Data are presented as the average accompanied by the standard error of the mean (SEM). Statistical analyses were carried out in Systat software. Statistical comparisons were done with a Student's t-test or, where appropriate, ANOVA followed by modified Bonferroni test comparisons; significance was defined as $p<0.05$.

\section{Results}

To expand the tools available to elucidate the molecular mechanisms underlying sleep and plasticity, we created a modified version of a Drosophila visual learning assay that has similarities to the Morris Water Maze $[17,18]$. The assay uses a "thermal maze" consisting of a $5 \times 5$ grid of Peltier plates maintained at $36-37^{\circ} \mathrm{C}$ (which is aversive to flies) (Figure 1). The first trial begins when a single tile that is associated with a specific visual cue is cooled to $25^{\circ} \mathrm{C}$. For subsequent trials, the cold tile is heated, the visual panorama is rotated and the flies must find the new cold tile by remembering its association with the visual cue. Over the course of 10 trials, flies get progressively faster at locating this cool spot [17].

To validate our modified Spatial Learning apparatus, we evaluated behavior in Canton-S (Cs) and 2U flies. Cs and 2U flies are frequently used as wild-type strains in sleep and memory studies, respectively [24-26]. As seen in Figure 2, A-F, sleep characteristics of $2 \mathrm{U}$ and $\mathrm{Cs}$ flies were in the range observed for wildtype flies [27] (Figure 2, A-F). To evaluate learning, an individual fly is placed into the apparatus and the time to find the cool spot is calculated. Learning during subsequent trials, expressed as the time to target, is normalized to the time to find the cold spot in the first trial. As seen in Figure 2, G and U, flies reduced their time to target by $\sim 80 \%$ over 10 trails, consistent with previous observations [17]. To evaluate the robustness of this assay in our lab, we evaluated learning in an independent cohort of $2 \mathrm{U}$ flies and found similar results (Figure 2, I). To simplify comparisons, we calculate a learning index (1 - [average relative time to target in trials $9 \& 10]$ ) $* 100$. As seen in Figure 2, $\mathrm{K}$, the two independent replicates of learning in $2 \mathrm{U}$ flies were not statistically different even though the experiments were conducted on separate cohorts of flies evaluated weeks apart. Importantly, Cs flies showed similar learning profiles and this pattern of behavior was also observed in an independent cohort (Figure 2, H, $\mathrm{J}$, and L). Thus, both $2 \mathrm{U}$ and Cs flies get progressively faster at locating the cool spot.

Although these data are consistent with previous results and suggest that flies are learning the location of the cool tile in relation to a visual cue, the flies may be using other cues to improve the speed with which they can escape the heated tiles that are not dependent on learning the association between the visual panorama and the location of the cold tile (e.g. self-motion cues, undetectable thermal gradients, etc.). To address this possibility, we uncoupled the visual cues from the location of the cool spot as previously described [17]. Specifically, the visual cues remained fixed while the cool spot location was changed. If flies in our assay were using nonvisual cues for learning, they should progressively reduce their time to target even in this uncoupled condition. However, we find that in contrast to the coupled condition, Cs flies did not get faster at finding the cold spot over time (Figure 2, M-O). Thus, flies in our assay use the distal visual cues to get progressively faster at locating the "cool spot."

\section{Learning is sleep dependent}

Sleep loss and extended waking result in cognitive deficits in a variety of tasks in animals from flies to humans [3, 28, 29]. We, therefore, hypothesized that sleep deprivation would also impair Spatial Learning. To test this hypothesis, we sleep-deprived Cs flies overnight using the Sleep Nullifying Apparatus [20], which deprived flies of $>98 \%$ of their sleep (Figure 3, A), and evaluated learning immediately following sleep deprivation. As we expected, sleep deprivation impaired learning compared with agematched controls (Figure 3, B). Sleep deprivation does not alter simple visual behaviors such as object fixation and optomotor responses [30], suggesting that this was a learning defect rather than impaired visual acuity. To investigate whether sleep deprivation can independently alter heat avoidance, we placed flies in a chamber in which one half was heated at $36-37^{\circ} \mathrm{C}$ and the other half was maintained at $25^{\circ} \mathrm{C}$. As seen in Figure 3, C, sleep deprivation did not alter heat avoidance compared with untreated, age-match controls. Thus, the deficits in Spatial Learning following sleep deprivation are not due to alterations in sensory thresholds.

\section{Enhancing sleep restores learning to rutabaga mutant flies}

The adenyl cyclase rutabaga (rut) was first identified as one of the canonical olfactory memory mutants in the fly [31]. rut mutants have since been shown to be impaired in a number of different learning assays [32-35]. Consequently, we hypothesized that rut mutants would also be impaired in Spatial Learning. As previously described, rut ${ }^{2080}$ mutants, which are in a Cs background, sleep the same as Cs controls $[19,36]$ (Figure 4, A). Despite having similar sleep profiles, rut ${ }^{2080}$ mutants displayed severe learning impairments (Figure 4, B). rut ${ }^{2080}$ mutants are not impaired in optomotor responses [37] and exhibit normal heat avoidance (Figure 4, C). Thus, rut ${ }^{2080}$ mutants display deficits in Spatial Learning.

Enhancing sleep pharmacologically, by administering the GABA-A agonist Gaboxadol, can restore learning in rut ${ }^{2080}$ mutants when evaluated using a variety of learning assays including (1) Aversive Phototaxis Suppression assay, (2) courtship conditioning, and (3) place learning $[19,36]$. To determine whether enhanced sleep could also restore Spatial Learning to rut $^{2080}$ mutants, we increased sleep in rut ${ }^{2080}$ mutants for 2 days by feeding them $0.1 \mathrm{mg} / \mathrm{mL}$ Gaboxadol $[19,38]$. As shown previously, Gaboxadol-fed rut ${ }^{2080}$ males show a robust increase 


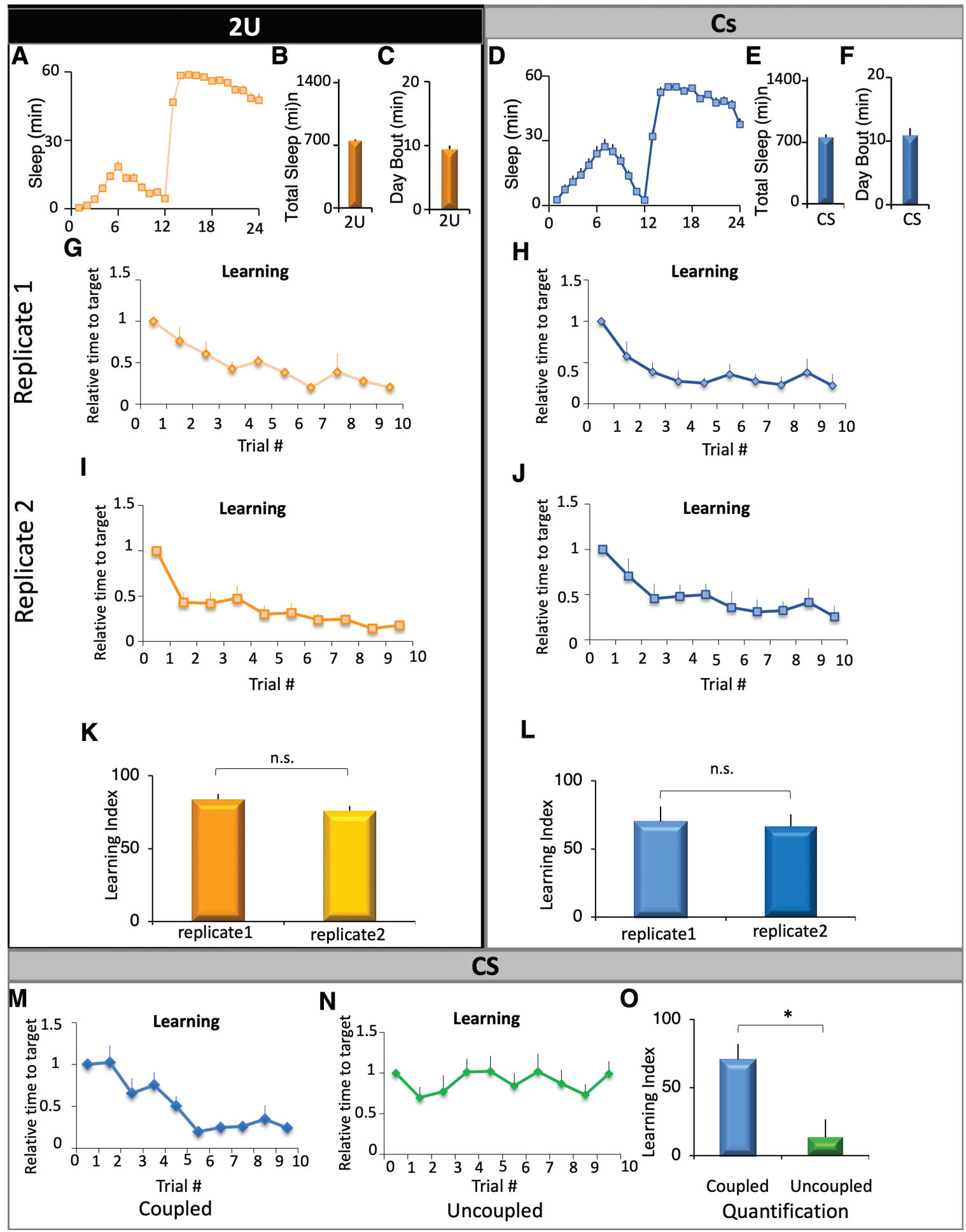

Figure 2. Validation of our spatial learning apparatus. (A) Sleep in minutes per hour for the 2U wild-type strain maintained on a 12:12 light-dark schedule (LD) ( $n=29$ flies). (B) Total sleep time in minutes in $2 \mathrm{U}$ flies. (C) Average daytime sleep bout duration (a measure of sleep consolidation during the day) in $2 \mathrm{U}$ flies. (D) Sleep in minutes per hour for the Cs wild-type strain maintained on a 12:12 LD schedule ( $n=32$ flies). (E) Total sleep time in minutes in Cs flies. (F) Average daytime sleep bout duration in Cs flies. (G and I) Spatial Learning in two independent cohorts of $2 \mathrm{U}$ flies trained in the coupled condition. Spatial Learning is expressed as the "time to target" normalized to the time in the first trial. Flies reduced their "time to target" over 10 trials by $~ 80 \%$ ( $n=9-11$ flies/replicate, repeated measures ANOVA for trials, $\left.F_{[9.162]}=15.36, p<10^{-10}\right)$. (K) Quantification of learning scores in G and I, expressed as percentage change in the time to target in trials 9 and 10 , relative to trial 1 . The two replicates of $2 \mathrm{U}$ flies exhibited similar Learning Indices (n.s. $p=0.09$, two-tailed t-test). (H and J) Learning in two independent replicates of $\mathrm{Cs}$ flies trained in the coupled condition. Flies reduced their "time to target" over 10 trials by $\sim 70 \%\left(n=8-10\right.$ flies/replicate, repeated measures ANOVA for trials $\left.F_{[9,160]}=12.28, p<10^{-12}\right)$. $(\mathrm{L})$ Two independent cohorts of Cs flies exhibited similar Learning Indices (n.s. $p=0.84$, two-tailed $t$-test). (M and N) In contrast to flies trained in the coupled condition, Cs flies in the uncoupled condition showed little to no improvement in their time to target ( $n=7-12$ flies/condition, two-way repeated-measures ANOVA for condition $\times$ trial $\left.F_{[9,198]}=4.93, p \leq 0.01\right)$. (O) Learning index of flies in the "coupled" condition is much higher than in the "uncoupled" condition $\left({ }^{*} p<0.01, t\right.$-test). 


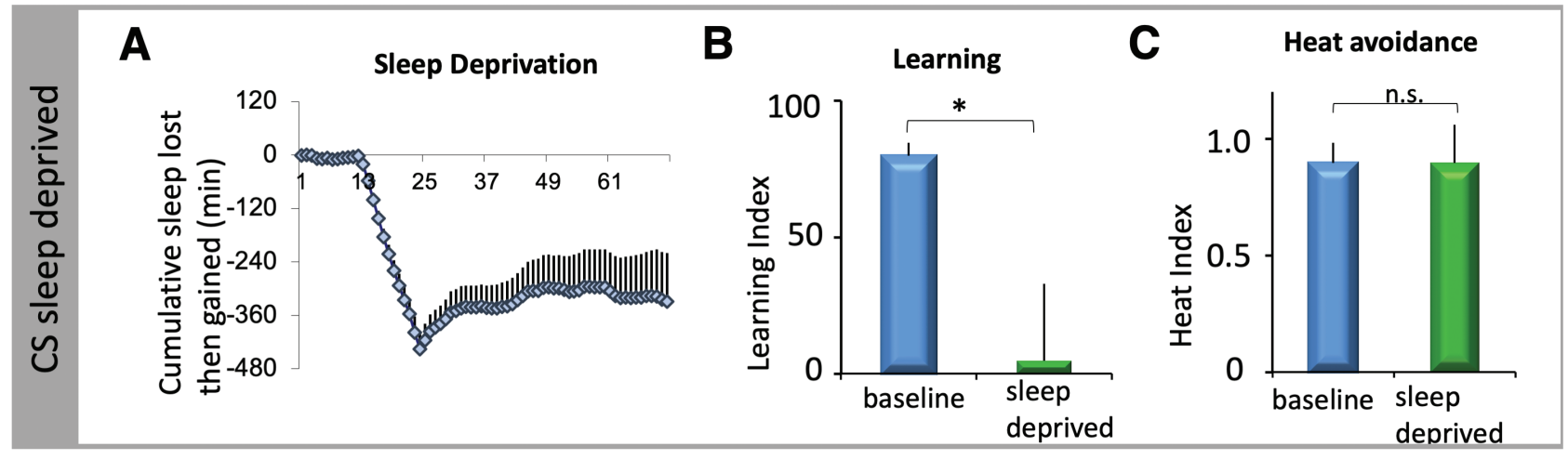

Figure 3. Sleep deprivation impairs Spatial Learning in Cs flies. (A) Sleep-deprived Cs flies lost $98 \%$ of their sleep and recovered $~ 60 \%$ of their lost sleep during the subsequent $48 \mathrm{~h}$ in recovery $\left(n=30\right.$ flies, repeated measures ANOVA for time, $\left.F_{[70,1470]}=12.97, p<10^{-15}\right)$. (B) Sleep-deprived Cs flies (green) display impaired Spatial Learning compared with controls (blue) ( $n=7-10$ flies/condition, ${ }^{*} p<0.01, t$-test). (C) Sleep deprivation did not impair heat avoidance ( $n=10-11 \mathrm{flies} / \mathrm{condition}, \mathrm{n} . \mathrm{s}$. $p=0.97, \mathrm{t}$-test).

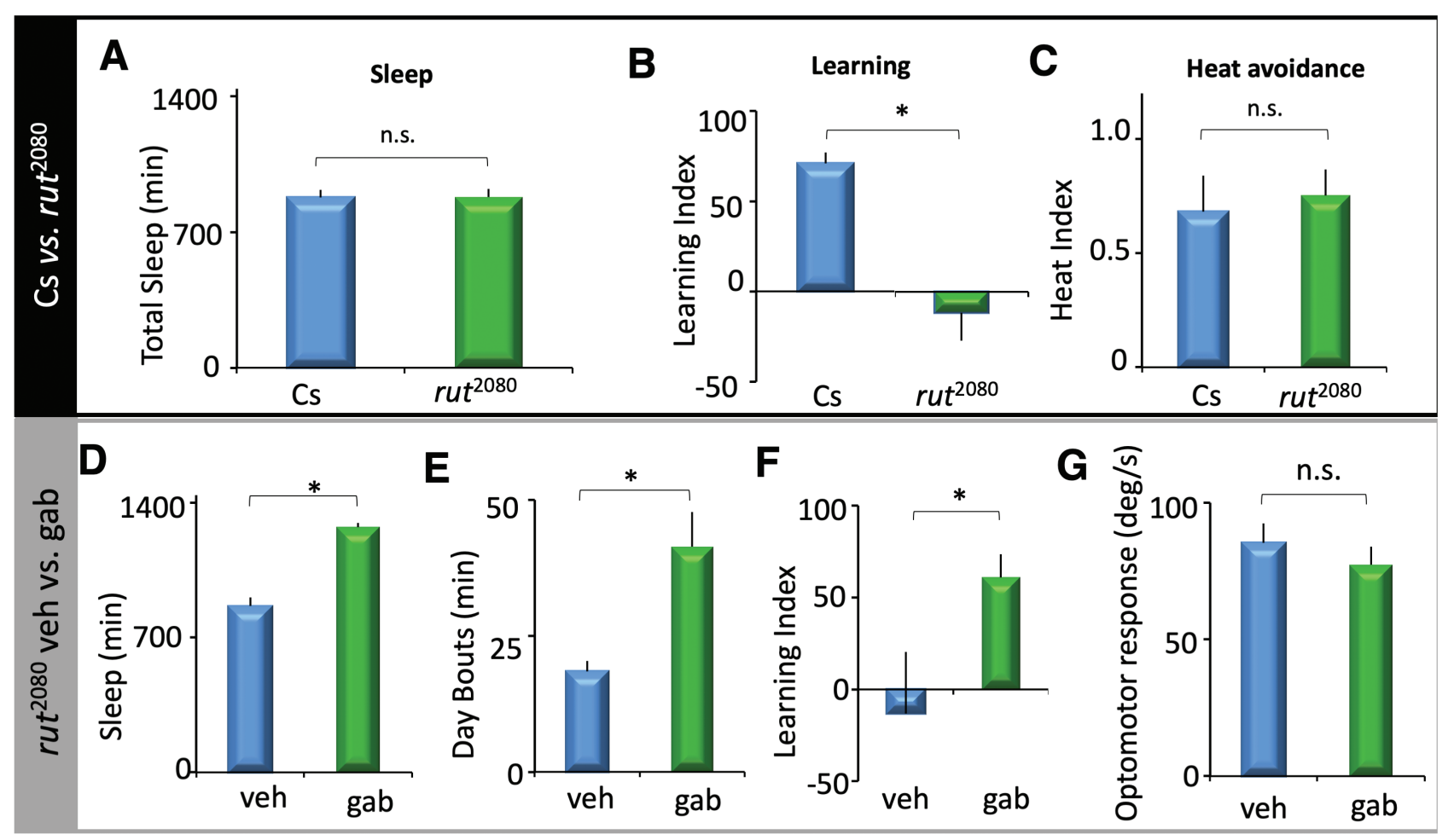

Figure 4. rut-dependent impairments in spatial learning are reversed by enhancing sleep. (A) Sleep profiles of rut ${ }^{2080}$ mutants was not different compared with Cs controls ( $n=18-22$ flies/genotype, n.s. $p=0.49$, t-test). (B) Spatial Learning is impaired in rut ${ }^{2080}$ mutants compared with Cs controls ( $n=9-10$ flies/genotype, ${ }^{*} p<0.001$, t-test). (C) rut ${ }^{2080}$ males are not impaired in heat avoidance ( $n=10$ flies/genotype, n.s. $\left.p=0.27\right)$. (D) Gaboxadol increases total sleep in rut ${ }^{2080}$ flies compared with vehiclefed siblings ( $n=18-20$ flies/condition, ${ }^{*} p<10^{-10}$, t-test). (E) Gaboxadol-fed rut ${ }^{2080}$ flies display increased average daytime sleep bout duration compared with vehicle-fed siblings $\left({ }^{*} p<10^{-4}\right.$, t-test). (F) Gaboxadol restored spatial learning to rut $^{2080}$ flies compared with controls $\left(n=9-10\right.$ flies/condition, ${ }^{*} p<0.01$, $t$-test). (G) Gaboxadol did not impair the optomotor response of $r u t^{2080}$ flies ( $n=19$ flies/condition, n.s. $p=0.43$, t-test).

in total sleep time which is accompanied by a significant increase in the average duration of sleep bouts during the day (a measure of sleep consolidation) (Figure 4, D and E). Gaboxadolfed rut ${ }^{2080}$ males also exhibited increased sleep duration and unaltered sleep bout length at night (data not shown). Importantly, Gaboxadol-induced sleep significantly improved the learning index compared with age-matched, vehicle-fed siblings; Gaboxadol did not alter optomotor behavior (Figure 4, F and G). Taken together, these results support and extend our previous observations that enhancing sleep can reverse the learning impairments in the classic memory mutant rut [19].
Age-dependent learning impairments are reversed by enhancing sleep

Age-dependent decline in cognitive performance has been observed in flies and humans [39-42]. In flies, some plasticity deficits are observed as early as 18-20 days of age [39, 42]. We, therefore, hypothesized that flies would exhibit age-dependent degradation in Spatial Learning as well. We started by examining sleep in 21-24 days old flies. As previously described, older flies had less total sleep and shorter average sleep bout duration than 4-5 days old flies (Figure 5, A-C) [24]. Importantly, waking activity of old flies was not altered indicating that locomotor 


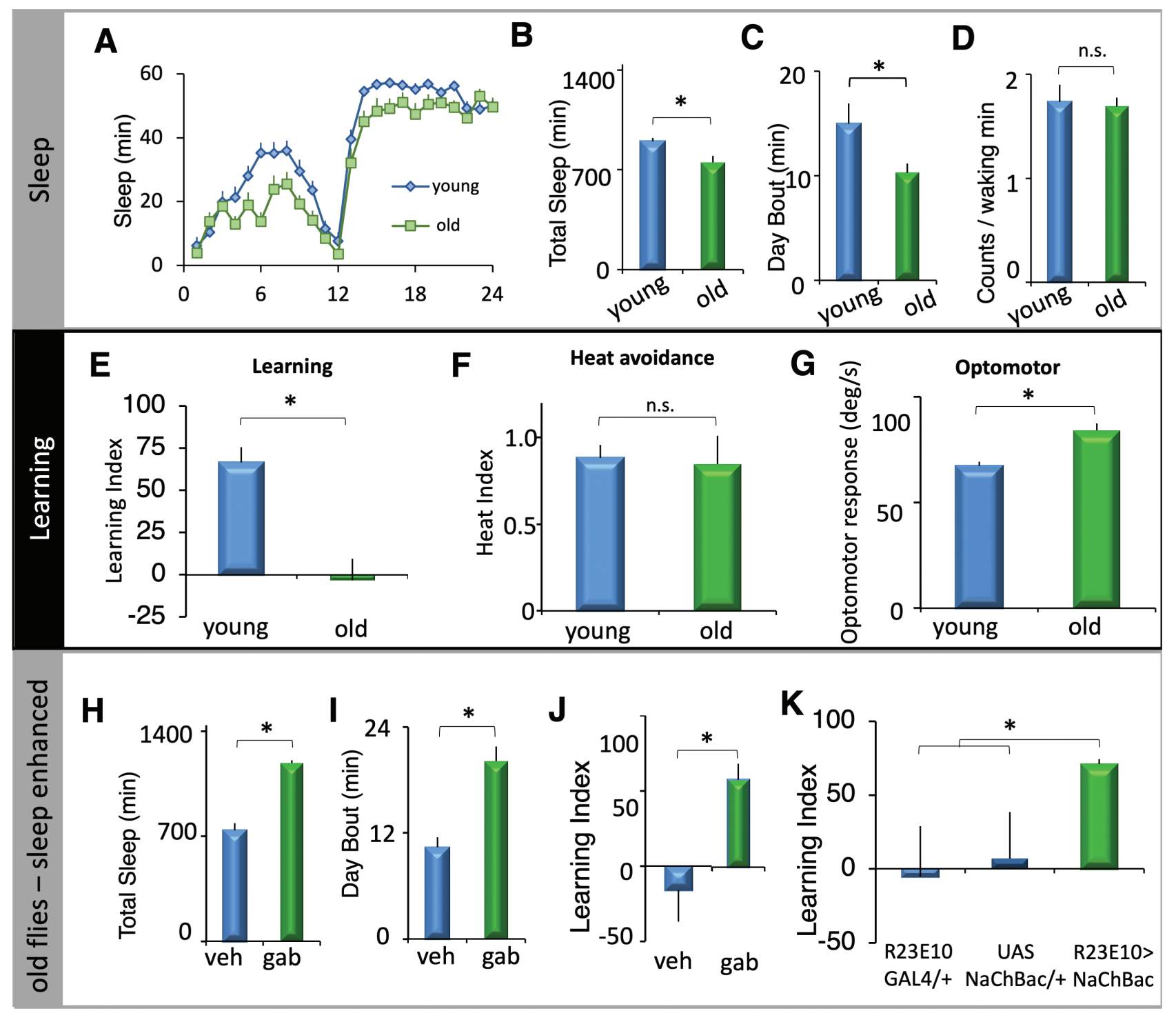

Figure 5. Age-dependent declines in spatial learning can be reversed by enhancing sleep. (A) Sleep, in minutes per hour, was reduced in 21-24 days old flies (green) compared with 5-day-old controls (blue) ( $n=20-27$ flies/group, repeated measures ANOVA age $\times$ time; $\left.F_{[23,966]}=5.49, p<0.001\right)$. (B) Total sleep was reduced in 21-24 days old flies compared with 5 -day-old flies; ${ }^{*} p<0.01$, t-test. (C) Aging reduced average daytime sleep bout duration; $p<0.05, t$-test. (D) Waking activity was not impaired in 21-24 days old flies (n.s. $p=0.83$ ). (E) Spatial learning was impaired in 21-24 days old flies compared with 5-day-old flies $\left(n=10-14\right.$ flies/group, $\left.{ }^{*} p<10^{-4}\right)$. (F) Age did not disrupt heat avoidance $(n=10$ flies/condition, n.s. $p=0.49)$. (G) Age did not impair optomotor responses $\left(n=51-62\right.$ flies/condition, $\left.{ }^{*} p<10^{-5}\right)$. (H) Gaboxadol $(G a b)$ increased sleep in 21-24 days old flies ( $n=20$ flies/group, ${ }^{*} p<10^{-10}$ ). (I) Gaboxadol increased average daytime sleep bout duration in $21-25$ days old flies (green) compared with age-matched controls (blue) $\left({ }^{*} p<10^{-4}\right.$, t-test). (J) Spatial learning was restored to Gaboxadol-fed 21-24 days old flies (green) compared with age-matched vehiclefed controls $\left(n=9-10\right.$ flies/condition, ${ }^{*} p<10^{-4}$, t-test). (K) Spatial learning was significantly higher in 21-24 days old R23E10-GAL4/+>UAS-NaChBac/+ flies compared with age-matched R23E10-GAL4/+ and UAS-NaChBac/+parental controls $\left(n=8-10\right.$ flies/genotype, One-way ANOVA for genotype $F_{[2,49 \mid}=4.59, p<0.05,{ }^{*} p<0.01$, modified Bonferroni test).

activity was not impaired (Figure 5, D). Interestingly, 21-24 days old flies displayed impairments in Spatial Learning compared with 4-5 days old flies (Figure 5, E). As above, the changes in performance were not associated with impairments in heat avoidance (Figure 5, F). Old flies exhibited an elevated optomotor response relative to young flies, that is, they are more sensitive to the stimulus (Figure 5, G). Importantly, although young and old flies do indeed differ, old flies are not impaired in optomotor behavior indicating that the degraded performance in Spatial Learning is not due to visual impairments. Taken together with our results above that aging does not alter heat avoidance, these data indicate that the learning defects we observed in old flies were not a consequence of defective sensory processing. Thus, 20-day-old flies also show deficits in Spatial Learning.

Given that older flies show sleep deficits (Figure 5, A-C) and previous reports have found that genetically enhanced sleep can restore plasticity to older flies, we hypothesized that enhancing sleep might also restore Spatial Learning to 20-24 days old flies [42]. We tested this hypothesis by enhancing sleep in old flies with two different methods-pharmacologically, by feeding old flies Gaboxadol and genetically, by activating the fan-shaped body (a known sleep center) [43]. Gaboxadol robustly increased sleep amount and consolidation in old flies (Figure 5, H and I). Crucially, Gaboxadol-enhanced sleep restored memory to 20-day-old flies 
compared with vehicle-fed, age-matched siblings (Figure 5, J). Gaboxadol did not alter heat avoidance in 20-day-old flies (see later section) indicating that the improvements were not due to changes in sensory thresholds. To confirm these results, sleep was increased by expressing the bacterial sodium channel $\mathrm{NaChBac}[44,45]$ under the control of the R23E10-GAL4 driver [46]. Consistent with previous results, activating the fan-shaped body increased sleep (data not shown). Importantly, 20-day-old R23E10-GAL4/+>UAS-NaChBac/+ flies displayed significantly higher learning scores than both parental controls (R23E10GAL4/+ and UAS-NaChBac/+) (Figure 5, K). Thus, inducing sleep with two independent methods reverses the age-dependent cognitive deficits we see using Spatial Learning. These results support previous suggestions that sleep can be used as a therapeutic to reverse age-dependent cognitive deficits $[47,48]$.

\section{Spatial Learning requires dopamine signaling}

The neuromodulator dopamine plays key roles in facilitating synaptic mechanisms that support learning and memory in flies and mammals [20, 49-52]. However, the potential role of dopaminergic signaling in Spatial Learning in flies has not yet been investigated. Thus, we evaluated Spatial Learning while using both pharmacology and genetics to modulate dopamine. We disrupted dopamine pharmacologically by feeding flies the dopamine synthesis inhibitor 3-Iodo L Tyrosine (3IY) [53]. As seen in Figure 6, A-C, feeding flies 3IY increased both total sleep time, and sleep consolidation during the day compared with age-matched vehicle-fed siblings; no impairments in waking activity were observed indicating that the flies were not motorically impaired (Figure 6, D). Together, these data highlight the wake-promoting effects of dopamine [54]. Importantly, feeding flies 3IY impaired learning (Figure 6, E-G), suggesting that Spatial Learning requires dopaminergic signaling.

To disrupt dopamine genetically, we inhibited most dopaminergic neurons by expressing the inwardly rectifying potassium channel KCNJ2 (UAS-Kir2.1 [55]) using Tyrosine Hydroxylase GAL4 (TH-GAL4). To confine the inhibition of dopaminergic neurons to the adult stage, and obtain better temporal control of inhibition, we used the TARGET system. The TARGET system uses a temperature-sensitive GAL4-suppressor, GAL80 ${ }^{\text {ts }}$. GAL80 is inactivated, thereby relieving the suppression of GAL4, and allowing the expression of UAS-Kir2.1 only at $30^{\circ} \mathrm{C}$ [56]. Flies were maintained at $30^{\circ} \mathrm{C}$ for 2 days prior to testing (Figure $6, \mathrm{H}$ and $\mathrm{J}$ ). Importantly, at $30^{\circ} \mathrm{C}, \mathrm{TH}$ GAL4 > GAL80's; UAS Kir 2.1 flies displayed both increased sleep (Figure 6, I), and impaired Spatial Learning (Figure 6, K) compared with siblings maintained at $18^{\circ} \mathrm{C}$; TH-GAL4/+ and tubP GAL80 ${ }^{\text {ts }}$, UAS Kir/+ parental lines displayed normal sleep and memory at $18^{\circ} \mathrm{C}$ and $30^{\circ} \mathrm{C}$. Thus, reducing dopamine levels with two different methods impairs Spatial Learning indicating that dopaminergic signaling is required for learning in this assay. Dopamine deficient flies have normal optomotor responses, visual fixation, and electroretinograms indicating that the Spatial Learning impairments were not a consequence of aberrant sensory processing [57].

\section{Enhancing dopamine signaling reverses age-dependent cognitive impairments}

Dopamine levels are known to decrease with age in flies, even as dopaminergic neurons appear to be anatomically unaffected [58, 59]. Further, we have previously shown that enhancing dopamine signaling in 20-day-old flies restores structural age-dependent deficits in behavioral plasticity [42]. Therefore, we hypothesized that enhancing dopamine signaling would reverse the age-dependent Spatial Learning impairments observed above. Dopamine was increased by feeding flies the dopamine precursor Levodopa (L-Dopa) [60, 61]. As seen in Figure 7, A, feeding 20-day-old flies L-DOPA disrupted nighttime sleep as previously reported [62, 63]. Importantly, Spatial Learning was restored in 20-day-old, L-Dopa-fed flies compared with their age-matched vehiclefed siblings (Figure 7, B). Importantly, feeding L-Dopa or Gaboxadol did not alter heat avoidance (Figure 7, C). Spatial Learning in flies is known to require the function of R1 ellipsoid body (EB) ring neurons [17]. Further, dopamine receptors are known to be expressed in the EB $[64,65]$. Combined with our results above showing that we could restore learning to aged flies by elevating dopamine levels, we hypothesized that age-dependent learning impairments could be reversed by elevating dopamine signaling in the EB. To test this hypothesis, we expressed the Drosophila Dopamine D1 Receptor (Dop1R1) in the EB using R15B07-GAL4. The Dop1R1 mutant, $\mathrm{dumb}^{2}$, contains a piggyBac inserted into the first intron of the Dop1R1 gene that contains a UAS that can be used to induce expression of a functional Dop1R1 receptor [66]. As seen in Figure 7, D, disruptions in Spatial Learning are reversed in 20-day-old R15B07-GAL4/+>dumb $2 /+$ flies compared with age-matched parental controls (15B07-GAL4/+ and dumb2/+). Thus, increasing dopaminergic signaling through the Dop1R1, specifically in the R1 ellipsoid body ring neurons rescues agedependent cognitive decline in Spatial Learning.

\section{Discussion}

We find that sleep plays an important role in supporting Spatial Learning in flies. Sleep deprivation impaired learning; conversely, enhancing sleep reversed learning impairments associated with rut mutants and aging. These data build on previous results that suggested a surprising restorative depth to the relationship between sleep and plasticity [19, 36], and extend them to a novel Spatial Learning task. As discussed below, our results are consistent with findings in rodent Spatial Learning and human episodic memory research, reinforcing the parallels between Spatial Learning in animal models and human declarative memories $[15,16]$.

The modified Spatial Learning assay studied here was adapted from previous designs $[17,18]$. In the published protocols, 8-10 replicates using 15 flies per replicate are tested using a between-subject design. In contrast, we chose to study individual flies to allow us to track learning in each fly using a within-subject design. Testing groups of 15-100 flies/replicate is standard in Drosophila learning and memory studies and provides many advantages [31,67]. However, testing individual flies in a within-subject design provides additional opportunities. For example, by studying the learning behavior of individual flies, we can isolate and study flies that display a range of phenotypes (e.g. different rates of learning). We have previously used this approach to identify genes that convey resilience or vulnerability to sleep loss [68]. Further, evaluating individual flies substantially reduces the computational power needed to evaluate details of behavior (path length, etc.). 


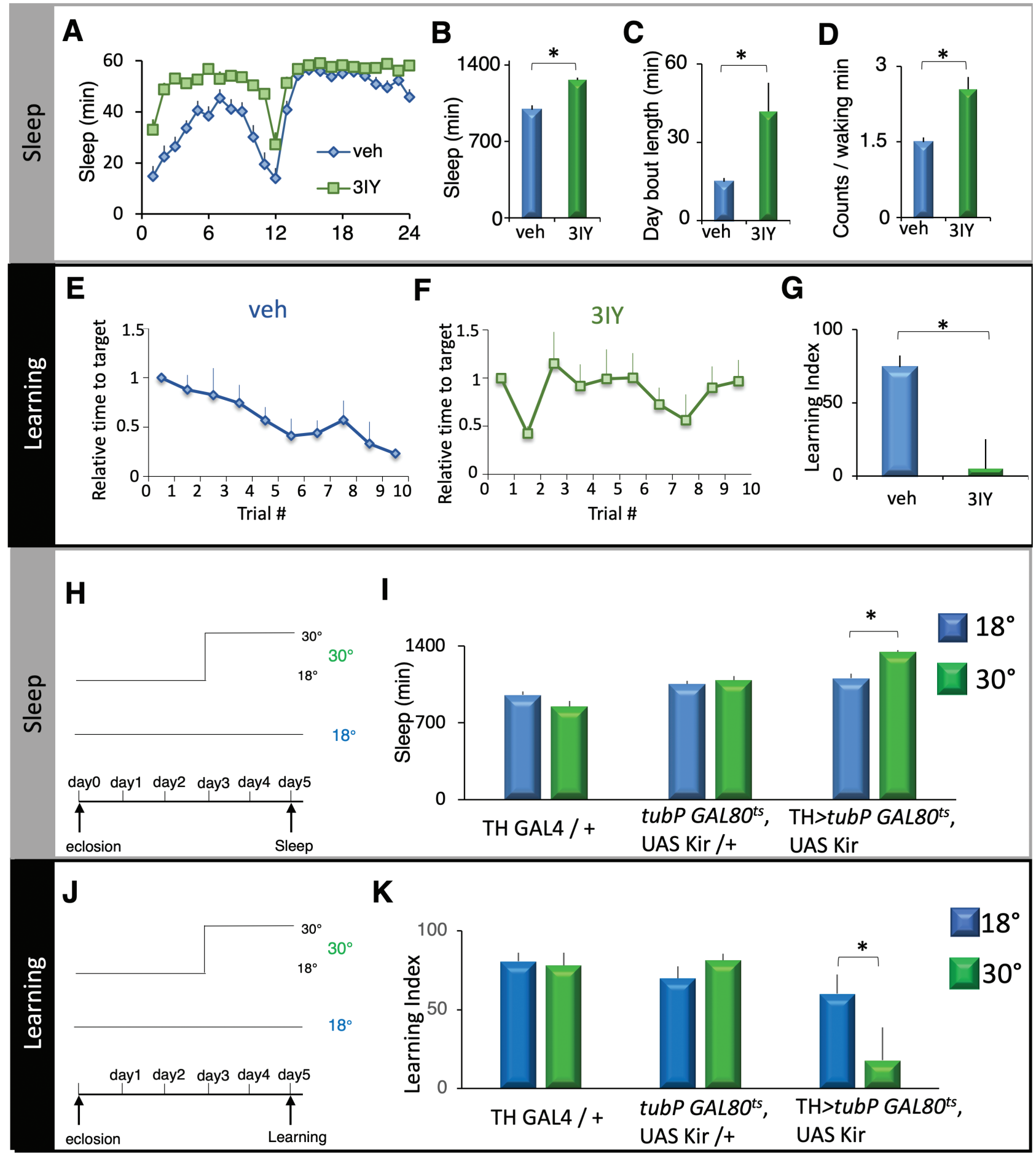

Figure 6. Reducing dopamine signaling impairs learning. (A) 3-Iodo-L-tyrosine (3IY)-fed flies display an increase in sleep compared with vehicle-fed controls (Sleep in minutes per hour, $n=20-21$ flies/group, two-way repeated-measures ANOVA for drug $\times$ time $F_{[23,782]}=5.49, p<10^{-6}$ ). (B) $3 I Y$-fed flies display more total sleep than age-matched vehicle-fed siblings $\left({ }^{*} p<10^{-6}\right.$, t-test). (C) 3IY increased average daytime sleep bout duration ( ${ }^{*} p<0.001$, $t$-test). (D) 3IY did not impair waking activity compared with vehicle-fed controls ( ${ }^{*} p<0.01$, t-test). (E) Vehicle-fed Cs controls displayed spatial learning. (F) In contrast to vehicle-fed flies, 3IY-fed Cs flies were impaired in spatial learning. ( $n=8$ flies/group, two-way ANOVA drug $\times$ trial, $\left.F_{[9,126]}=2.33, p<0.05\right)$. (G) Learning index of 3IY-fed flies was greatly reduced compared with vehicle-fed controls $\left({ }^{*} p<0.01\right.$, t-test). (H) Schematic of temperature-shift experiment for sleep. Sleep is recorded over $24 \mathrm{~h}$ of 5 -day-old TH-GAL4/+, tubpGAl80 ${ }^{\text {ts }}$, UAS $\mathrm{Kir} /+$, and TH-GAL4/+ > tubpGAl80 ts , UAS Kir/+ flies maintained at $18^{\circ} \mathrm{C}$ and of their sibling flies that are reared for 3 days at $18^{\circ} \mathrm{C}$, and then shifted to the elevated temperature of $30^{\circ} \mathrm{C}$ for 2 days prior to testing. (I) TH-GAL4/+>GAL80 s, UAS Kir/+ flies displayed an increase in sleep at $30^{\circ} \mathrm{C}$ compared with siblings maintained at $18^{\circ} \mathrm{C}$; sleep in TH-GAL4/+ or the tubP GAL80 ${ }^{\text {ts }}$, UAS Kir/+ parental controls was similar at both $18^{\circ} \mathrm{C}$ and $30^{\circ} \mathrm{C}(n=20-30$ flies/group, two-way ANOVA for genotype $\times$ temperature, $F_{[2,131]}=7.28, p<0.01 ;{ }^{*} p<0.001$, modified Bonferroni test). (J) Schematic of temperature-shift experiment for learning. Spatial learning is evaluated in 5 -day-old TH-GAL4/+, tubpGAl80 ts , UAS Kir/+, and TH-GAL4/+ > tubpGAl80 ${ }^{\text {ts }}$, UAS Kir/+ flies maintained at $18^{\circ} \mathrm{C}$ and of their sibling flies that are reared for 3 days at $18^{\circ} \mathrm{C}$, and then shifted to the elevated temperature of $30^{\circ} \mathrm{C}$ for 2 days prior to testing. (K) Spatial learning is impaired in $\mathrm{TH}>\mathrm{GAL} 80^{\mathrm{ts}}$, UAS Kir flies at $30^{\circ} \mathrm{C}$ compared with siblings maintained at $18^{\circ} \mathrm{C}$; temperature did not impact spatial learning in either TH-GAL4/+ or the tubP GAL80 ${ }^{\text {ts }}$, UAS Kir/+ parental controls ( $n=8-12$ flies/group, Two-way ANOVA for genotype $\times$ temperature, $F_{[2,116]}=4.96, p<0.01,{ }^{*} p<0.05$, modified Bonferroni test). 


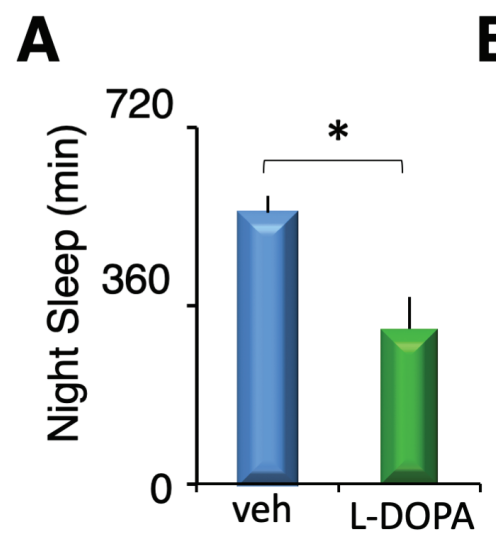

B

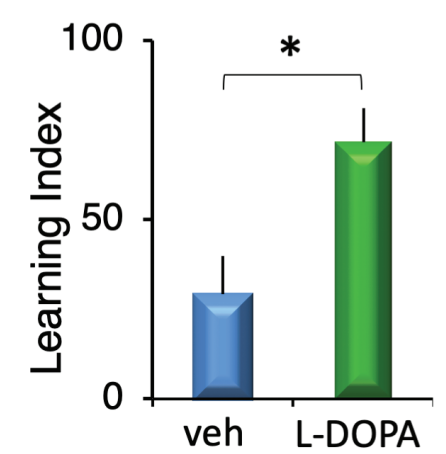

C

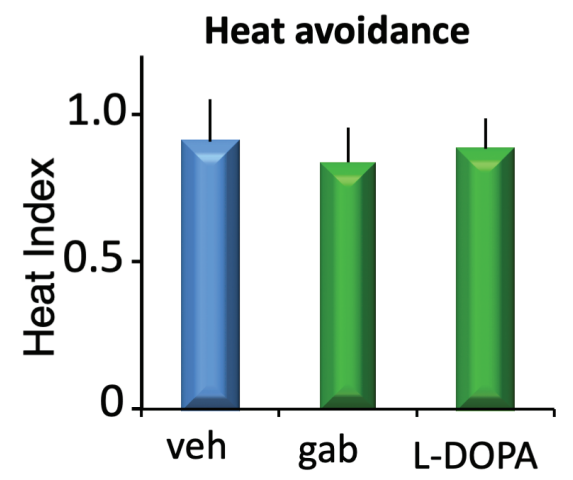

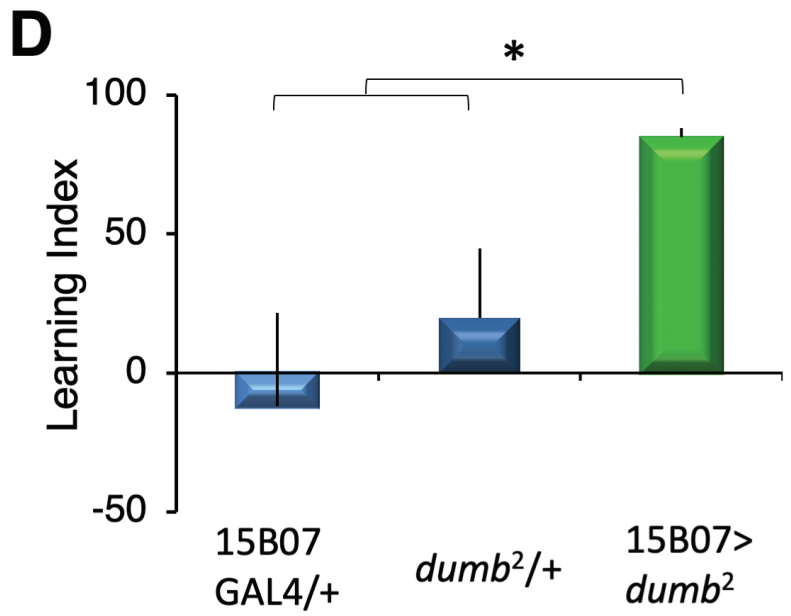

Figure 7. Enhancing dopamine signaling ameliorates age-related cognitive decline. (A) Nighttime sleep was reduced in levodopa (L-Dopa, green) fed 21-24 days old Cs flies compared with age-matched vehicle-fed (blue) controls ( $n=10-12$ flies/group, ${ }^{*} p<0.01$, t-test). (B) Spatial learning was elevated in old L-Dopa fed Cs flies (green) compared with age-matched controls (blue) ( $n=9-10$ flies/group, $\left.{ }^{*} p<0.01\right)$. (C) Heat avoidance was not changed in old flies fed L-Dopa or Gaboxadol (green) compared with age-matched controls ( $n=10$ flies/condition, n.s. $p>0.25$, modified Bonferroni test). (D) Spatial learning was elevated in old R15B07>dumb flies compared with age-matched R15B07-GAL4/+ and dumb $2 /+$ parental controls ( $n=8$ flies/genotype, One-way ANOVA for genotype $F_{[2,45]}=5.82, p<0.01 ;{ }^{*} p<0.01$, planned comparisons, modified Bonferrsoni test).

Moreover, testing individual flies also reduces the amount of time required to generate the necessary flies to complete a given experiment and can expedite discovery experiments. Importantly, examining Spatial Learning in 8-10 flies/genotype produces statistically robust datasets. Indeed, significant learning was observed in two independent replicates of two different wild-type strains-Cs and $2 \mathrm{U}$, thus validating our design for Spatial Learning.

\section{Spatial Learning is sleep dependent}

Depriving flies of sleep overnight impaired Spatial Learning. These results are consistent with experiments in rodents and humans. In rodents, sleep deprivation impaired encoding of hippocampus-dependent spatial memory as assessed with the Morris Water Maze, while sleep loss had minimal effects on hippocampus-independent nonspatial tasks [69, 70]. These experiments in rodents are corroborated by studies in humans that found that human spatial memory was dependent on sleep [71]. Further, sleep deprivation in humans also impaired learning in declarative memory tasks which are known to require hippocampus function $[12,13]$.
Interestingly, Kirszenblat et al. found that sleep deprivation impaired visual selective attention in flies [30]. Our data suggest that the two assays are likely measuring different aspects of behavior. For example, Kirszenblat et al. demonstrated that rut mutants are deficient in selective attention and that Gaboxadolinduced sleep was unable to restore this deficit [30]. However, as we report here, Gaboxadol-induced sleep can restore Spatial Learning to rut mutants. Since Gaboxadol-induced sleep cannot "fix" selective attention in rut mutants but can restore performance in Spatial Learning, these data indicate that, as measured by these two methods, visual attention and Spatial Learning are dissociable. In this regard, it is worth noting that Kirszenblat et al. also found that flies require $24 \mathrm{~h}$ of sleep deprivation to disrupt selective attention while $12 \mathrm{~h}$ of sleep deprivation was without effect [30]. Interestingly, we find deficits in Spatial Learning after $12 \mathrm{~h}$ of sleep deprivation.

\section{Enhancing sleep restores Spatial Learning to rut mutants}

The rutabaga (rut) mutant was first isolated as one of the canonical fly learning and memory mutants using olfactory 
conditioning [31]. rut mutants have since been shown to be impaired in a number of different learning and memory assays, and have been used to validate new assays [20, 31, 3335]. We, therefore, evaluated Spatial Learning in rut mutants. Indeed, we find that rut mutants do not exhibit any sleep defects, but nonetheless, are severely impaired in Spatial Learning. Moreover, enhancing sleep pharmacologically by feeding rut mutant flies Gaboxadol for 2 days restored Spatial Learning. These results are consistent with previous work showing that Gaboxadol-enhanced sleep restored learning to rut mutants in other learning assays: Aversive Phototaxis Suppression, courtship conditioning, and place learning $[19,36]$. Importantly, rut mutants do not display sleep defects during baseline. As a consequence, it is unlikely that Gaboxadol-induced sleep is simply ameliorating preexisting sleep deficiencies. Rather, the enhanced sleep induced by Gaboxadol is likely to exert its effects on neuronal plasticity in memory circuits. For olfactory conditioning, rut has been proposed to function as a coincidence detector in mushroom body Kenyon cells, detecting coincident input of the conditioned stimulus (odor) and the unconditioned stimulus (electric shock) [72]. However, rut is widely expressed, and likely functions as a signaling molecule in multiple cellular processes to influence different aspects of neural plasticity [19, $37,73-78]$. Indeed, it should be noted that the brain processes odors using sparse coding and that, during olfactory conditioning, the electric shock is very precisely timed with brief puffs of odors to induce a lasting association [31, 79]. In contrast, flies evaluated using many operant learning assays, such as courtship conditioning, place learning, and Aversive Phototaxic Suppression, experience a more continuous exposure to the aversive stimulus (quinine, mate-rejection, and heat) [32, 80-82]. In any event, the precise role of rut in Spatial Learning, its site of action, and the mechanism by which enhanced sleep restores learning to rut mutants remain unknown and are the subject of ongoing study.

\section{Enhancing sleep reverses age-dependent cognitive decline}

Age-related memory impairments are observed in humans, and appear to disproportionately affect hippocampus-dependent episodic memories and spatial memory [83-85]. Further, aging is also accompanied by sleep deficits and defects in sleepdependent memory consolidation [5, 86]. Enhancing sleep in older adults was also able to ameliorate age-related impairments $[47,48]$.

Flies too have been shown to exhibit age-dependent cognitive decline $[39,40,42,87]$. In some cases, plasticity deficits have been observed at 18-20 days of age [39, 42]. Further enhancing sleep in aged flies by feeding Gaboxadol reversed age-dependent defects in social enrichment-induced plasticity [42]. Consistent with these results, we found that 20-24 days old flies were impaired in Spatial Learning and that enhancing sleep could reverse these impairments. Interestingly, the agedependent Spatial Learning impairments we observed appear to be more severe than those observed with olfactory conditioning [39]. These data parallel studies in rodents that found that aging impaired hippocampus-dependent Spatial Learning but did not appear to affect hippocampus-independent nonSpatial Learning [88-90].

\section{Dopamine signaling is required for Spatial Learning}

We find that inhibiting dopaminergic signaling with two different methods increased sleep and impaired Spatial Learning. In rodents, dopamine secreted from locus coeruleus to the hippocampus also plays a critical role in mediating spatial Learning [91-93]. Our data thus support a conserved role for dopamine in Spatial Learning, consistent with its role as a key facilitator of synaptic plastic changes that support learning and memory [49-51].

It is worth noting that both methods of inhibiting dopaminergic signaling increased sleep while impairing learning. We have argued previously that a thorough characterization of sleep should not rely exclusively on examining sleep metrics only. Healthy sleep promotes a number of positive nonsleep variables such as memory, plasticity, metabolism, immune function, etc. Determining whether a change in sleep induced by a genetic manipulation impacts these other variables is essential for understanding whether sleep has been positively or negatively impacted $[28,68]$. Our data clearly indicate that the increased sleep associated with impaired dopaminergic signaling is associated with impairments in Spatial Learning. Given that Gaboxadol-induced sleep restores Spatial Learning to rut ${ }^{2080}$ mutants and 20-21 days old flies, we hypothesize that disrupting dopamine signaling disrupts sleep efficiency. Indeed, while dumb ${ }^{2}$ flies sleep more, they are also more arousable at night, suggesting they are not sleeping as deeply [94]. That is, the flies would need to sleep more to compensate for this ineffective sleep.

Another nonexclusive hypothesis is that different subsets of dopaminergic neurons support arousal and Spatial Learning. The fly arousal promoting dopaminergic neurons are known to project to the fan-shaped body and the mushroom body [95-97]. The dopaminergic neurons that support Spatial Learning are not yet known. Our Spatial Learning assay requires the function of EB ring neurons [17]. Dopamine receptors are known to be expressed in the EB $[64,65]$. Further, dopaminergic neurons have been described that project into the EB $[65,98]$ from the PPM3 cluster. These PPM3 EB projecting dopaminergic neurons are promising candidates for mediating Spatial Learning. Thus, the effects of dopaminergic inhibition on sleep and learning could map to different subsets of dopaminergic neurons. Further experiments are needed to distinguish between these possibilities. Nonetheless, the apparent discord between increasing sleep and impairing learning serves to highlight the importance of functional evaluation of sleep outcomes when describing manipulations that affect sleep time [28].

\section{Enhancing dopaminergic signaling reverses age- dependent impairment}

Complementary to the experiments above where we inhibited dopaminergic signaling, we find that increasing dopaminergic signaling restored Spatial Learning to aged flies. These results are consistent with previous work in flies and mammals. Dopamine levels decline with age in flies and humans [58, 99, 100]. Further, enhancing dopaminergic signaling reversed aspects of age-dependent cognitive decline. In humans, elevating dopaminergic signaling ameliorated age-dependent declines in episodic memory [101]. Similarly, increasing dopaminergic signaling restored Spatial Learning in rodents [90], and reversed age-dependent defects in social enrichment-induced 
plasticity in flies [42]. How is enhancing dopaminergic signaling able to restore learning to aged brains? Overexpressing the Dop1R1 dopamine receptor in EB ring neurons restored learning to aged flies. This result suggests the possibility that aging disrupts dopaminergic signaling to the $\mathrm{EB}$ (at the level of dopamine release and/or receptivity). This possibility will be addressed in future work.

\section{Conclusions}

Collectively our results demonstrate a critical role for sleep in supporting Spatial Learning in flies. Sleep deprivation impairs Spatial Learning. Conversely, enhancing sleep restores learning to impaired brains. Our data are consistent with work on Spatial Learning in rodents and spatial and episodic memories in humans, indicating that the phenomena we report are conserved. Interestingly, Spatial Learning in mammals is dependent on the hippocampus, and is closely associated with the phenomenon of replay. When animals are trained to run along a linear track, their trajectories are represented by a sequence of activation of hippocampal place cells [102]. These sequences are replayed in a time-compressed fashion during sleep [103-105], in a complex dialog between the hippocampus and the cortex [106-109], to consolidate the memory of the experience [110, 111]. Further, these place cell sequences can be reactivated by cueing in sleep [112-114]. Although replay-like phenomena have not been observed in flies, cued reactivation during sleep improved recall in bees [115], and reactivation during sleep of dopaminergic neurons involved in memory acquisition was shown to facilitate consolidation of courtship memory in flies [116], suggesting that such replay-like processes might be detected in Drosophila too.

\section{Acknowledgments}

We thank Lijuan Cao for technical assistance, Michael Reiser for helpful advice and technical input, and Matt Thimgan and Stephane Dissel for a critical reading of the manuscript.

\section{Funding}

This work was supported by National Institutes of Health [grants 5R01NS051305-14 and 5R01NS076980-08 to P.J.S.].

\section{Disclosure statements}

Financial disclosure: none.

Non-financial disclosure: none.

\section{References}

1. Rechtschaffen A. Current perspectives on the function of sleep. Perspect Biol Med. 1998;41(3):359-390.

2. Dissel S, et al. Sleep and memory formation in Drosophila. In: Byrne JH, ed. Learning and Memory: A Comprehensive Reference. 2nd ed. Cambridge, MA: Academic Press; 2017:517-527.

3. Walker MP, et al. Sleep, memory, and plasticity. Annu Rev Psychol. 2006;57:139-166.
4. Stickgold R, et al. Sleep-dependent memory triage: evolving generalization through selective processing. Nat Neurosci. 2013;16(2):139-145.

5. Mander BA, et al. Sleep and human aging. Neuron. 2017;94(1):19-36.

6. Lucey BP, et al. How amyloid, sleep and memory connect. Nat Neurosci. 2015;18(7):933-934.

7. Fischer $\mathrm{S}$, et al. Sleep forms memory for finger skills. Proc Natl Acad Sci USA. 2002;99(18):11987-11991.

8. Walker MP, et al. Practice with sleep makes perfect: sleepdependent motor skill learning. Neuron. 2002;35(1):205-211.

9. Ellenbogen JM, et al. Human relational memory requires time and sleep. Proc Natl Acad Sci USA. 2007;104(18):7723-7728.

10. Gais $\mathrm{S}$, et al. Low acetylcholine during slow-wave sleep is critical for declarative memory consolidation. Proc Natl Acad Sci USA. 2004;101(7):2140-2144.

11. Diekelmann S, et al. The memory function of sleep. Nat Rev Neurosci. 2010;11(2):114-126.

12. Yoo SS, et al. A deficit in the ability to form new human memories without sleep. Nat Neurosci. 2007;10(3):385-392.

13. Drummond SP, et al. Altered brain response to verbal learning following sleep deprivation. Nature. 2000;403(6770):655-657.

14. Nestor PJ, et al. Declarative memory impairments in Alzheimer's disease and semantic dementia. Neuroimage. 2006;30(3):1010-1020.

15. Buzsáki G. Theta rhythm of navigation: link between path integration and landmark navigation, episodic and semantic memory. Hippocampus. 2005;15(7):827-840.

16. Buzsáki G, et al. Memory, navigation and theta rhythm in the hippocampal-entorhinal system. Nat Neurosci. 2013;16(2):130-138.

17. Ofstad TA, et al. Visual place learning in Drosophila melanogaster. Nature. 2011;474(7350):204-207.

18. Foucaud J, et al. Use of spatial information and search strategies in a water maze analog in Drosophila melanogaster. PLoS One. 2010;5(12):e15231.

19. Dissel $\mathrm{S}$, et al. Sleep restores behavioral plasticity to Drosophila mutants. Curr Biol. 2015;25(10):1270-1281.

20. Seugnet L, et al. D1 receptor activation in the mushroom bodies rescues sleep-loss-induced learning impairments in Drosophila. Curr Biol. 2008;18(15):1110-1117.

21. Shaw PJ, et al. Stress response genes protect against lethal effects of sleep deprivation in Drosophila. Nature. 2002;417(6886):287-291.

22. Colomb J, et al. Open source tracking and analysis of adult Drosophila locomotion in Buridan's paradigm with and without visual targets. PLoS One. 2012;7(8):e42247.

23. Straw AD. Vision egg: an open-source library for realtime visual stimulus generation. Front Neuroinform. 2008;2:4.

24. Shaw PJ, et al. Correlates of sleep and waking in Drosophila melanogaster. Science. 2000;287(5459):1834-1837.

25. Hendricks JC, et al. A non-circadian role for cAMP signaling and CREB activity in Drosophila rest homeostasis. Nat Neurosci. 2001;4(11):1108-1115.

26. Tubon TC Jr, et al. dCREB2-mediated enhancement of memory formation. J Neurosci. 2013;33(17):7475-7487.

27. Andretic $R$, et al. Essentials of sleep recordings in Drosophila: moving beyond sleep time. Methods Enzymol. 2005;393:759-772.

28. Dissel S, et al. Sleep, performance, and memory in Flies. Curr Sleep Med Rep. 2015;1(1):47-54.

29. Van Dongen HP, et al. The cumulative cost of additional wakefulness: dose-response effects on neurobehavioral 
functions and sleep physiology from chronic sleep restriction and total sleep deprivation. Sleep. 2003;26(2):117-126.

30. Kirszenblat L, et al. Sleep regulates visual selective attention in Drosophila. J Exp Biol. 2018;221(Pt 24):jeb191429.

31. Tully $\mathrm{T}$, et al. Classical conditioning and retention in normal and mutant Drosophila melanogaster. J Comp Physiol A. 1985;157(2):263-277.

32. Seugnet L, et al. Aversive phototaxic suppression: evaluation of a short-term memory assay in Drosophila melanogaster. Genes Brain Behav. 2009;8(4):377-389.

33. Diegelmann $\mathrm{S}$, et al. Genetic dissociation of acquisition and memory strength in the heat-box spatial learning paradigm in Drosophila. Learn Mem. 2006;13(1):72-83.

34. O'Dell KM, et al. Abnormal courtship conditioning in males mutant for the RI regulatory subunit of Drosophila protein kinase A. J Neurogenet. 1999;13(1-2):105-118.

35. Pan Y, et al. Differential roles of the fan-shaped body and the ellipsoid body in Drosophila visual pattern memory. Learn Mem. 2009;16(5):289-295.

36. Dissel S, et al. Sleep restores place learning to the adenylyl cyclase mutant rutabaga. J Neurogenet. 2020;34(1):83-91.

37. van Swinderen B.Attention-like processes in Drosophila require short-term memory genes. Science. 2007;315(5818):1590-1593.

38. Dissel S, et al. Enhanced sleep reverses memory deficits and underlying pathology in Drosophila models of Alzheimer's disease. Neurobiol Sleep Circadian Rhythms. 2017;2:15-26.

39. Tamura T,etal.Agingspecificallyimpairsamnesiac-dependent memory in Drosophila. Neuron. 2003;40(5):1003-1011.

40. Rieche F, et al. Drosophila full-length amyloid precursor protein is required for visual working memory and prevents age-related memory impairment. Curr Biol. 2018;28(5):817823.e3.

41. Bishop NA, et al. Neural mechanisms of ageing and cognitive decline. Nature. 2010;464(7288):529-535.

42. Donlea JM, et al. Genetic rescue of functional senescence in synaptic and behavioral plasticity. Sleep. 2014;37(9):1427-1437.

43. Donlea $\mathrm{JM}$, et al. Inducing sleep by remote control facilitates memory consolidation in Drosophila. Science. 2011;332(6037):1571-1576.

44. Luan $\mathrm{H}$, et al. Functional dissection of a neuronal network required for cuticle tanning and wing expansion in Drosophila. J Neurosci. 2006;26(2):573-584.

45. Nitabach MN, et al. Electrical hyperexcitation of lateral ventral pacemaker neurons desynchronizes downstream circadian oscillators in the fly circadian circuit and induces multiple behavioral periods. J Neurosci. 2006;26(2):479-489.

46. Donlea JM, et al. Neuronal machinery of sleep homeostasis in Drosophila. Neuron. 2014;81(4):860-872.

47. Papalambros NA, et al. Acoustic enhancement of sleep slow oscillations and concomitant memory improvement in older adults. Front Hum Neurosci. 2017;11:109.

48. Westerberg CE, et al. Memory improvement via slowoscillatory stimulation during sleep in older adults. Neurobiol Aging. 2015;36(9):2577-2586.

49. Cognigni $P$, et al. Do the right thing: neural network mechanisms of memory formation, expression and update in Drosophila. Curr Opin Neurobiol. 2018;49:51-58.

50. Pignatelli $\mathrm{M}$, et al. Role of dopamine neurons in reward and aversion: a synaptic plasticity perspective. Neuron. 2015;86(5):1145-1157.

51. Aso Y, et al. The neuronal architecture of the mushroom body provides a logic for associative learning. Elife. 2014;3:e04577.
52. Schwaerzel M, et al. Dopamine and octopamine differentiate between aversive and appetitive olfactory memories in Drosophila. J Neurosci. 2003;23(33):10495-10502.

53. Neckameyer WS. Multiple roles for dopamine in Drosophila development. Dev Biol. 1996;176(2):209-219.

54. Andretic R, et al. Dopaminergic modulation of arousal in Drosophila. Curr Biol. 2005;15(13):1165-1175.

55. Baines RA, et al. Altered electrical properties in Drosophila neurons developing without synaptic transmission. $J$ Neurosci. 2001;21(5):1523-1531.

56. McGuire SE, et al. Spatiotemporal rescue of memory dysfunction in Drosophila. Science. 2003;302(5651):1765-1768.

57. Riemensperger $\mathrm{T}$, et al. Behavioral consequences of dopamine deficiency in the Drosophila central nervous system. Proc Natl Acad Sci USA. 2011;108(2):834-839.

58. Neckameyer WS, et al. Dopamine and senescence in Drosophila melanogaster. Neurobiol Aging. 2000;21(1):145-152.

59. White $\mathrm{KE}$, et al. The dopaminergic system in the aging brain of Drosophila. Front Neurosci. 2010;4:205.

60. Budnik V, et al. Genetic dissection of dopamine and serotonin synthesis in the nervous system of Drosophila melanogaster. J Neurogenet. 1987;4(6):309-314.

61. Livingstone MS, et al. Genetic dissection of monoamine neurotransmitter synthesis in Drosophila. Nature 1983;303(5912):67-70.

62. Aboudhiaf S, et al. LAT1-like transporters regulate dopaminergic transmission and sleep in Drosophila. Sleep. 2018;41(10). doi: 10.1093/sleep/zsy137.

63. Pfeiffenberger $\mathrm{C}$, et al. Cul3 and the BTB adaptor insomniac are key regulators of sleep homeostasis and a dopamine arousal pathway in Drosophila. PLoS Genet. 2012;8(10):e1003003.

64. Li W, et al. microRNA-276a functions in ellipsoid body and mushroom body neurons for naive and conditioned olfactory avoidance in Drosophila. J Neurosci. 2013;33(13):5821-5833.

65. Kong EC, et al. A pair of dopamine neurons target the D1-like dopamine receptor DopR in the central complex to promote ethanol-stimulated locomotion in Drosophila. PLoS One. 2010;5(4):e9954.

66. Kim YC, et al. D1 dopamine receptor dDA1 is required in the mushroom body neurons for aversive and appetitive learning in Drosophila. J Neurosci. 2007;27(29):7640-7647.

67. Kahsai L, et al. Learning and memory in Drosophila: behavior, genetics, and neural systems. Int Rev Neurobiol. 2011;99:139-167.

68. Dissel S, et al. Differential activation of immune factors in neurons and glia contribute to individual differences in resilience/vulnerability to sleep disruption. Brain Behav Immun. 2015;47:75-85.

69. Guan Z, et al. Sleep deprivation impairs spatial memory and decreases extracellular signal-regulated kinase phosphorylation in the hippocampus. Brain Res. 2004;1018(1):38-47.

70. Hairston IS, et al. Sleep restriction suppresses neurogenesis induced by hippocampus-dependent learning. Neurophysiol. 2005;94(6):4224-4233.

71. Ferrara M, et al. Sleep to find your way: the role of sleep in the consolidation of memory for navigation in humans. Hippocampus. 2008;18(8):844-851.

72. Gervasi N, et al. PKA dynamics in a Drosophila learning center: coincidence detection by rutabaga adenylyl cyclase and spatial regulation by dunce phosphodiesterase. Neuron. 2010;65(4):516-529. 
73. Engel JE, et al. Altered habituation of an identified escape circuit in Drosophila memory mutants. J Neurosci. 1996;16(10):3486-3499.

74. Paranjpe P, et al. Gustatory habituation in Drosophila relies on rutabaga (adenylate cyclase)-dependent plasticity of GABAergic inhibitory neurons. Learn Mem. 2012;19(12):627-635.

75. Berke B, et al. Retrograde BMP signaling at the synapse: a permissive signal for synapse maturation and activitydependent plasticity. J Neurosci. 2013;33(45):17937-17950.

76. Renger JJ, et al. Role of cAMP cascade in synaptic stability and plasticity: ultrastructural and physiological analyses of individual synaptic boutons in Drosophila memory mutants. J Neurosci. 2000;20(11):3980-3992.

77. Donlea JM, et al. Use-dependent plasticity in clock neurons regulates sleep need in Drosophila. Science. 2009;324(5923):105-108.

78. Brembs B, et al. Double dissociation of PKC and AC manipulations on operant and classical learning in Drosophila. Curr Biol. 2008;18(15):1168-1171.

79. Hige $\mathrm{T}$, et al. Plasticity-driven individualization of olfactory coding in mushroom body output neurons. Nature. 2015;526(7572):258-262.

80. Siegel RW, et al. Conditioned responses in courtship behavior of normal and mutant Drosophila. Proc Natl Acad Sci USA. 1979;76(7):3430-3434.

81. Le Bourg E, et al. Learned suppression of photopositive tendencies in Drosophila melanogaster. Anim Learn Behav. 2002;30(4):330-341.

82. Putz G, et al. Memories in drosophila heat-box learning. Learn Mem. 2002;9(5):349-359.

83. Jennings JM, et al. An opposition procedure for detecting age-related deficits in recollection: telling effects of repetition. Psychol Aging. 1997;12(2):352-361.

84. Toth JP, et al. Effects of age on estimated familiarity in the process dissociation procedure: the role of noncriterial recollection. Mem Cognit. 2006;34(3):527-537.

85. Newman MC, et al. Spatial memory and aging: performance on a human analog of the Morris Water Maze. Aging, Neuropsychology, and Cognition. 2000;7(2):86-93.

86. Pace-Schott EF, et al. Age-related changes in the cognitive function of sleep. Prog Brain Res. 2011;191:75-89.

87. Mery F. Aging and its differential effects on consolidated memory forms in Drosophila. Exp Gerontol. 2007;42(1-2):99-101.

88. Rapp PR, et al. An evaluation of spatial information processing in aged rats. Behav Neurosci. 1987;101(1):3-12.

89. Barnes CA. Memory deficits associated with senescence: a neurophysiological and behavioral study in the rat. J Comp Physiol Psychol. 1979;93(1):74-104.

90. Bach ME, et al. Age-related defects in spatial memory are correlated with defects in the late phase of hippocampal long-term potentiation in vitro and are attenuated by drugs that enhance the cAMP signaling pathway. Proc Natl Acad Sci USA. 1999;96(9):5280-5285.

91. Xing B, et al. Dopamine D1 but not D3 receptor is critical for spatial learning and related signaling in the hippocampus. Neuroscience. 2010;169(4):1511-1519.

92. da Silva WC, et al. D1/D5 dopamine receptors modulate spatial memory formation. Neurobiol Learn Mem. 2012;97(2):271-275.

93. Kempadoo KA,et al.Dopamine release from the locus coeruleus to the dorsal hippocampus promotes spatial learning and memory. Proc Natl Acad Sci USA. 2016;113(51):14835-14840.
94. Faville R, et al. How deeply does your mutant sleep? Probing arousal to better understand sleep defects in Drosophila. Sci Rep. 2015;5:8454.

95. Liu $\mathrm{Q}$ et al. Two dopaminergic neurons signal to the dorsal fan-shaped body to promote wakefulness in Drosophila. Curr Biol. 2012;22(22):2114-2123.

96. Ueno $\mathrm{T}$, et al. Identification of a dopamine pathway that regulates sleep and arousal in Drosophila. Nat Neurosci. 2012;15(11):1516-1523.

97. Sitaraman D, et al. Control of sleep by dopaminergic inputs to the Drosophila mushroom body. Front Neural Circuits. 2015;9:73.

98. Liang $\mathrm{X}$, et al. Morning and evening circadian pacemakers independently drive premotor centers via a specific dopamine relay. Neuron. 2019;102(4):843-857.e4.

99. Bäckman L, et al. The correlative triad among aging, dopamine, and cognition: current status and future prospects. Neurosci Biobehav Rev. 2006;30(6):791-807.

100. Bäckman L, et al. Linking cognitive aging to alterations in dopamine neurotransmitter functioning: recent data and future avenues. Neurosci Biobehav Rev. 2010;34(5):670-677.

101. Chowdhury R, et al. Dopamine modulates episodic memory persistence in old age. J Neurosci. 2012;32(41):14193-14204.

102. Wilson MA, et al. Dynamics of the hippocampal ensemble code for space. Science. 1993;261(5124):1055-1058.

103. Wilson MA, et al. Reactivation of hippocampal ensemble memories during sleep. Science. 1994;265(5172):676-679.

104. Lee AK, et al. Memory of sequential experience in the hippocampus during slow wave sleep. Neuron. 2002;36(6):1183-1194.

105. Nádasdy Z, et al. Replay and time compression of recurring spike sequences in the hippocampus. J Neurosci. 1999;19(21):9497-9507.

106. Siapas AG, et al. Coordinated interactions between hippocampal ripples and cortical spindles during slowwave sleep. Neuron. 1998;21(5):1123-1128.

107. Sirota A, et al. Communication between neocortex and hippocampus during sleep in rodents. Proc Natl Acad Sci U S A. 2003;100(4):2065-2069.

108. Ji D, et al. Coordinated memory replay in the visual cortex and hippocampus during sleep. Nat Neurosci. 2007;10(1):100-107.

109. Rothschild G, et al. A cortical-hippocampal-cortical loop of information processing during memory consolidation. Nat Neurosci. 2017;20(2):251-259.

110. Girardeau G, et al. Selective suppression of hippocampal ripples impairs spatial memory. Nat Neurosci. 2009;12(10):1222-1223.

111. Ego-Stengel V, et al. Disruption of ripple-associated hippocampal activity during rest impairs spatial learning in the rat. Hippocampus. 2010;20(1):1-10.

112. Rasch B, et al. Odor cues during slow-wave sleep prompt declarative memory consolidation. Science. 2007;315(5817):1426-1429.

113. Bendor D, et al. Biasing the content of hippocampal replay during sleep. Nat Neurosci. 2012;15(10):1439-1444.

114. Vaz AP, et al. Replay of cortical spiking sequences during human memory retrieval. Science. 2020;367(6482):1131-1134.

115. Zwaka $\mathrm{H}$, et al. Context odor presentation during sleep enhances memory in honeybees. Curr Biol. 2015;25(21):2869-2874.

116. Dag $\mathrm{U}$, et al. Neuronal reactivation during post-learning sleep consolidates long-term memory in Drosophila. eLife. 2019;8:e42786. 